\title{
Phase state and velocity measurements with high temporal and spatial resolution during melting of $n$-octadecane in a rectangular enclosure with two heated vertical sides
}

\author{
J. Vogel*, D. Bauer \\ German Aerospace Center (DLR), Pfaffenwaldring 38-40, 70569 Stuttgart, Germany
}

J. Vogel, D. Bauer, Phase state and velocity measurements with high temporal and spatial resolution during melting of n-octadecane in a rectangular enclosure with two heated vertical sides, Int. J. Heat Mass Transf. 127 (2018) 1264 - 1276,

https://doi.org/10.1016/j.ijheatmasstransfer.2018.06.084.

\begin{abstract}
A novel validation experiment for the melting of a phase change material is presented. The goal is to measure phase state and velocities with high accuracy and resolution. The geometry and boundary conditions of the test section are the most generic found in latent heat storage systems: a phase change material is contained in a rectangular enclosure, where it is isothermally heated from two opposing vertical side walls. The enclosure has a height of $105 \mathrm{~mm}$ and a width and depth of $50 \mathrm{~mm}$. The bottom, front and back sides are solid transparent walls and on top is a thin layer of air. Near-adiabatic boundary conditions are realized at the non-heated sides with a surrounding insulated air-filled chamber and an actively controlled trace heating system. In this study n-octadecane is used as the phase change material. During melting, the liquid phase fraction is measured with a shadowgraph technique and velocities due to natural convection in the liquid phase are measured with particle image velocimetry (PIV). Interior and boundary temperatures are measured with thermocouples to control and analyze boundary effects. A thorough error estimation is done for all the measured quantities. The main result is a comprehensive dataset of liquid phase fractions and velocities with high spatial and temporal resolutions. The liquid phase fraction is additionally measured for three different driving temperature differences and a scaling by dimensionless numbers is performed. This results in a correlation function for the liquid phase fraction that predicts similar melting processes and is valuable in system design and optimization.
\end{abstract}

\section{Keywords}

Latent heat thermal energy storage; phase change material (PCM); natural convection; particle image velocimetry (PIV); validation experiment

\footnotetext{
* Corresponding author E-mail address: julian.vogel@dlr.de
} 


\begin{tabular}{|c|c|c|c|}
\hline \multicolumn{4}{|c|}{ Nomenclature } \\
\hline Latin & & Greek & \\
\hline$A$ & aspect ratio & $\beta$ & thermal expansion coefficient, $[\beta]=1 / \mathrm{K}$ \\
\hline$a$ & thermal diffusivity, $[a]=\mathrm{m}^{2} / \mathrm{s}$ & $\delta$ & distance of free surface from max. height, $[\delta]=\mathrm{m}$ \\
\hline$b_{1}, b_{2}$ & curve-fit function parameters & $\mu$ & dynamic viscosity, $[\mu]=\mathrm{Pa} \mathrm{s}$ \\
\hline$c$ & specific heat capacity, $[c]=\mathrm{J} /(\mathrm{kg} \mathrm{K})$ & $v$ & kinematic viscosity, $[v]=\mathrm{m}^{2} / \mathrm{s}$ \\
\hline$d$ & diameter, $[d]=\mathrm{m}$ & $\rho$ & density, $[\rho]=\mathrm{kg} / \mathrm{m}^{3}$ \\
\hline$D$ & depth, $[D]=\mathrm{m}$ & $\sigma$ & surface tension, $[\sigma]=\mathrm{N} / \mathrm{m}$ \\
\hline Fo & Fourier number & $\tau$ & characteristic time, $[\tau]=\mathrm{s}$ \\
\hline$f_{\mathrm{l}}$ & liquid phase fraction & & \\
\hline$g$ & gravity constant, $[g]=\mathrm{m} / \mathrm{s}^{2}$ & \multicolumn{2}{|c|}{ Subscripts } \\
\hline$H$ & height, $[H]=\mathrm{m}$ & 0 & initial value \\
\hline$k$ & thermal conductivity, $[k]=\mathrm{W} /(\mathrm{m} \mathrm{K})$ & $\mathrm{f}$ & flow \\
\hline$L$ & latent heat, $[L]=\mathrm{kJ} / \mathrm{kg}$ & $\mathrm{g}$ & gravitational \\
\hline$R a$ & Rayleigh number & 1 & liquid \\
\hline$R e$ & Reynolds number & $\mathrm{m}$ & melting point \\
\hline St & Stokes number & $\max$ & maximum \\
\hline Ste & Stefan number & $\min$ & minimum \\
\hline$T$ & temperature, $[T]={ }^{\circ} \mathrm{C}$ & $\mathrm{p}$ & particle \\
\hline$t$ & time, $[t]=\mathrm{s}$ & $\mathrm{S}$ & solid \\
\hline$\tilde{t}$ & scaled time & $\mathrm{w}$ & wall \\
\hline $\operatorname{Pr}$ & Prandtl number & \multirow{5}{*}{\multicolumn{2}{|c|}{$\begin{array}{l}\text { Symbols } \\
\Delta \quad \text { finite difference }\end{array}$}} \\
\hline$U$ & bulk velocity, $[U]=\mathrm{mm} / \mathrm{s}$ & & \\
\hline$v$ & velocity, $[v]=\mathrm{mm} / \mathrm{s}$ & & \\
\hline$W$ & width, $[W]=\mathrm{m}$ & & \\
\hline$x, y, z$ & coordinates, $[x, y, z]=\mathrm{m}$ & & \\
\hline
\end{tabular}

\section{Introduction}

Climate experts have been warning of the consequences of anthropogenic climate change for several decades. The Intergovernmental Panel on Climate Change states: "Warming of the climate system is unequivocal, and since the 1950s, many of the observed changes are unprecedented over decades to millennia. The atmosphere and ocean have warmed, the amounts of snow and ice have diminished, and sea level has risen" [1].

One of the most promising contributions to counteract climate change is the increased use of sustainable energy sources free of greenhouse gas emissions. The storage of thermal energy can play a critical role in improving the availability of fluctuating sustainable energy sources [2].

Latent heat storage (LHS) with solid-liquid phase change materials provides a high energy density with only a small temperature change near the phase change temperature. An overview of materials and applications has been given by Mehling and Cabeza [3] and Bauer et al. [4]. In combination with processes that use a heat transfer fluid that changes phase, such as steam generation or condensation, low overall temperature differences can be achieved. This leads to high exergetic efficiency, which is a suitable performance indicator of a LHS system [5].

Although latent heat storage has advantages over other storage technologies and research storage systems already exist, it is barely used in industrial applications. The main problem is the extensive engineering of a storage system. Every system must be uniquely adapted to the intended purpose, which leads to repetitive engineering work. This is why numerical simulations become increasingly important. With an appropriate numerical model, the storage system can be optimized without excessive testing. However, solid-liquid phase change is a complicated process that requires detailed transient modeling, especially when natural convection in the liquid phase is the dominant heat transfer mode. Although many models have been developed to simulate solid-liquid phase change, model validation is still an issue.

Detailed experiments of solid-liquid phase change are rare and many are not fully suitable for validation of numerical models. The state of research of melting experiments in rectangular enclosures with isothermal heating from the side is elaborated in section 2 of this article. To sum it up shortly, there is an urgent need for more detailed experiments [6]. There is also no validation experiment for the typical LHS design with symmetric heating from two opposite vertical side walls. Moreover, the velocity field in the resulting fully transient melting process has not yet been thoroughly measured.

Consequently, we developed a novel validation experiment specifically for a LHS system with symmetric heating from two opposite vertical side walls. An application-oriented storage system would have a complex geometry with heat transfer structures for increased power. However, a complicated geometry would restrict the measurement techniques; especially optical access to the PCM would not be 
possible. Therefore we chose a simplified geometry while keeping the principal topology of the LHS system. The simplest suitable geometries are the rectangular, the cylindrical or the spherical enclosure. We decided in favor of the rectangular enclosure, because it allows undistorted optical visibility of the phase front during melting and because it is commonly used in latent heat storage designs. An overview of melting in different types of enclosures is given in the review by Dhaidan et al. [7].

The result is a generic geometry with optimal measurement conditions that inherits the boundary conditions of a LHS system. Optical measurement techniques were applied: the phase state was measured by shadowgraphy and the velocities in the liquid phase were measured by particle image velocimetry (PIV). In this article we present data of phase front and velocity with high temporal and spatial resolution along with internal and boundary temperatures. We close our analysis by scaling results of the liquid phase fraction and propose a correlation function that predicts similar melting processes.

\section{State of research}

In the following, the state of research on melting in rectangular enclosures with isothermally heated vertical walls is summarized from the most relevant literature.

The typically investigated geometry has been a rectangular enclosure with an isothermally heated side wall and an opposing side that has been either isothermally cooled [6,8-14] or insulated $[15,16]$. The bottom has been an insulated wall and the top has been either a wall $[8,10,12-15]$ or a free surface $[6,9,11,17]$. The PCM has been initially in the solid state with a temperature below and near the melting point and then has been melted by raising the temperature of the heated wall to a constant value above the melting point.

Organic materials with low melting temperature such as paraffin [6], $n$-octadecane [8,9,11,15], $n$-heptadecane [17], lauric acid [16] or metals such as gallium $[10,13,14]$ or tin [12] have been used.

To reduce heat losses to the environment, all experiments have been insulated and some have additionally used a system of active trace heating to raise the temperature in a surrounding casing to the melting temperature of the PCM $[8,10]$.

Researchers have reported the formation of gas filled cavities during solidification that lead to bubbles rising throughout the melting process. The reason is that the investigated materials have a higher density in the solid phase than in the liquid phase and ambient air or other gases solved in the liquid phase fill the resulting cavities. To reduce this problem, it has been suggested to vibrate the cell [8] or solidify only small layers of material at once $[11,16]$.

To allow photographic observation of the phase front of materials that are transparent in the liquid form, transparent front and back walls made of acrylic glass have been used $[6,8,9,11,15-17]$. For opaque materials, the pour-out and probing method [10,12] and x-Ray radioscopy [13] have been used to measure the phase front. Both the phase front and velocities in the liquid phase in one direction at 5 distinct positions have been measured by ultrasonic Doppler velocimetry (UDV) [14].

By evaluating the phase front over time, the resulting temporal evolutions of the liquid phase fraction have been obtained $[6,10,12,15,16]$ and correlations have been discussed $[6,10,12]$. From the melting rate, the surfaceaveraged Nusselt number has been derived $[8-11,15,16]$. Some researchers also have calculated the Nusselt number locally over the height of the enclosure by evaluating horizontal slices, see for example [16].

Temperature measurements in the PCM by thermocouples have been done by many researchers $[6,8-11,13-16]$. With Interferometry, additional information about temperature isotherms and heat transfer at the heated wall and the solidliquid interface has been gained [17].

The main results are summarized as follows: Melting is mostly by heat conduction at the beginning, but natural convection predominates at later times with higher melting rates at the top than at the bottom, which leads to a curved phase front shape [8]. The transition point between conduction and convection [15] and the variation of heat transfer over the height [8] can be determined in dimensionless form. Dimensional analysis of the melting process reveals the four dimensionless groups Rayleigh number $R a$, Prandtl number $\operatorname{Pr}$, Stefan number Ste and aspect ratio $A$ [9]. The melting rate depends on the Rayleigh number, the aspect ratio and the dimensionless time, which is the product of Fourier number $F_{O}$ and Stefan number Ste. It is found that the melting rate decreases with increasing aspect ratio [10]. Natural convection is quasi-steady and not strongly influenced by the movement of the interface, because the flow velocities are much larger than the velocity of the interface movement [11]. The Nusselt number has a local maximum around the top of the solid PCM [16].

Despite the many findings from literature, there has been a strong interest in more experiments [6]. We identified the following experimental features or results that are still missing in the state of research: There is no validation experiment for the geometric arrangement with two opposite vertical sides that are isothermally heated while all other sides are adiabatic, although this design is common in latent heat storage $[18,19]$. The influence of boundaries is usually not assessed and boundary temperatures or heat flow rates are usually not published. Finally, there is no comprehensive data on flow velocities in the liquid phase, i.e. transient data with high spatial and temporal resolution.

\section{A novel validation experiment}

We developed a validation test case for melting in rectangular enclosures with initial and boundary conditions typically found in latent heat storage: the PCM was initially in the solid state at a temperature just below the melting point and then isothermally heated by two opposite vertical side walls until the liquid state is reached. The test bench was designed for an operation temperature range of $20 \ldots 250{ }^{\circ} \mathrm{C}$, however, in this article we present low-temperature measurements with $n$ - 
octadecane. Special care was taken to reduce boundary effects and analyze their impact on the experimental data. All the significant physical quantities, which are liquid phase fraction, velocities in the liquid phase due to natural convection and temperature were measured.

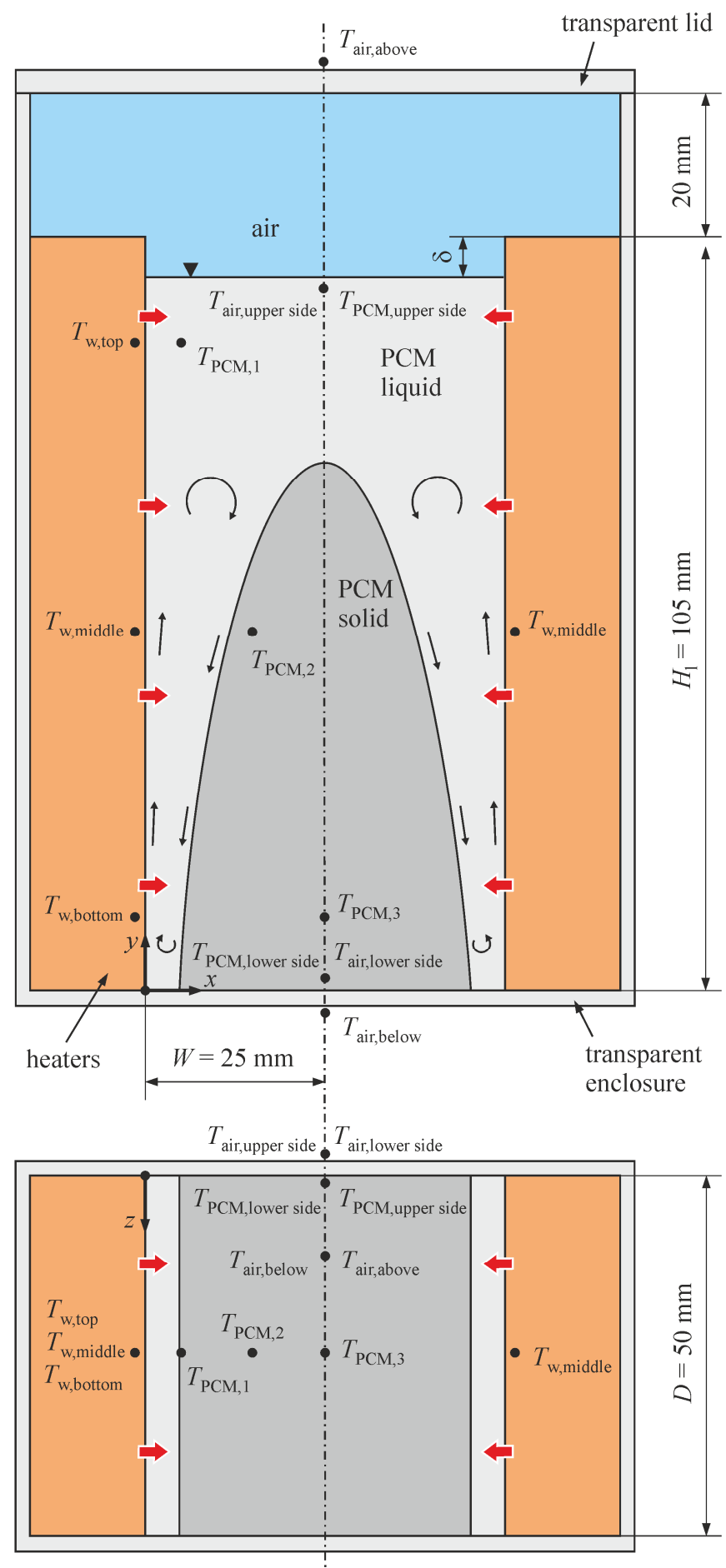

Figure 1: Test case definition with front view (upper image) and top view (lower image). Thermocouple positions are shown with filled circles.

\subsection{Test case definition}

The test case is illustrated in Figure 1: the rectangular test volume has a width of $50 \mathrm{~mm}$ between the two heated walls. Because of the symmetric arrangement, the relevant width for this test case is the half width $W=25 \mathrm{~mm}$. The height of the heated walls and the initial fill height of the PCM in the liquid state are $H_{1}=105 \mathrm{~mm}$. On top of the PCM, a small air layer allows volume expansion of the PCM and serves as first layer of insulation. During solidification the fill height of the PCM decreases by the length $\delta$ due to a larger density in the solid state. After solidification it is $\delta \approx 10 \mathrm{~mm}$ and the solid fill height is about $H_{\mathrm{s}}=95 \mathrm{~mm}$. The initial fill height in the liquid state was specifically chosen to achieve an average fill height of $H=100 \mathrm{~mm}$. The depth of the test volume $D=50 \mathrm{~mm}$ is large enough to reduce boundary effects at the mid-plane induced by the front and back walls and small enough to ensure a good quality of optical measurements.

The initial temperature $T_{0}$ is below the melting temperature $T_{\mathrm{m}}$ of the PCM. At time $t_{0}=0$, the wall temperature $T_{\mathrm{w}}$ is raised to a value $T_{\mathrm{w}}=T_{\mathrm{m}}+\Delta T$ to perform the melting process. In the ideal case, the bottom, front and back walls and the free surface at the top of the PCM would be adiabatic.

\subsection{Test chamber design and operation}

We realized the test case shown in Figure 1 with two heating plates placed in a transparent rectangular enclosure. For these low temperature experiments an enclosure made of acrylic glass PLEXIGLAS $®$ was used. We used custom built electric heaters made of stainless steel 1.4301. Each one was made of two plates that were welded together. A meandering path was milled into one of the plates to leave space for a heating coil that can heat the plates uniformly. To measure and control the wall temperature of the two heaters at mid-height $T_{\text {w,middle, two }}$ T-type thermocouples with $0.7 \mathrm{~mm}$ diameter were used. The thermocouples were positioned $1 \mathrm{~mm}$ inside the heating plates at the center of the heater wall. To test the temperature uniformity of the steel plates, the temperatures at the top $T_{\mathrm{w} \text {,top }}$ and bottom $T_{\mathrm{w} \text {,bottom }}$ of one of the heating plates were measured over a complete melting process. The heaters were fixed onto a lid construction for the transparent enclosure that also features two fused silica top windows for insulation and optical accessibility.

The transparent enclosure with the heaters and lid construction was placed into an outer chamber equipped with temperature controlled trace heaters to heat up the air and thus achieve near-adiabatic boundary conditions. A CAD drawing and a photograph of the test chamber are shown in Figure 2. The casing of the outer chamber was made of stainless steel 1.4301, in which fused silica windows were inserted at the sides. The outside faces were covered with an insulation material of $20 \mathrm{~mm}$ thickness with a thermal conductivity of $0.05 \mathrm{~W} /(\mathrm{m} \mathrm{K})$. Cutouts can be removed at the front and back sides and at the top to allow optical access, see Figure $2 \mathrm{~b}$ ). 


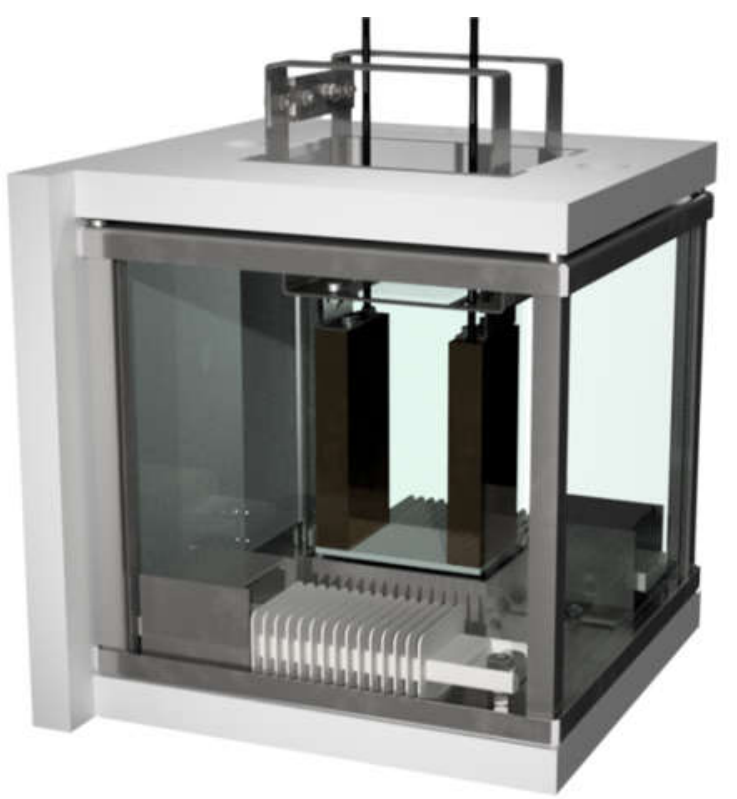

a) CAD drawing of test chamber design

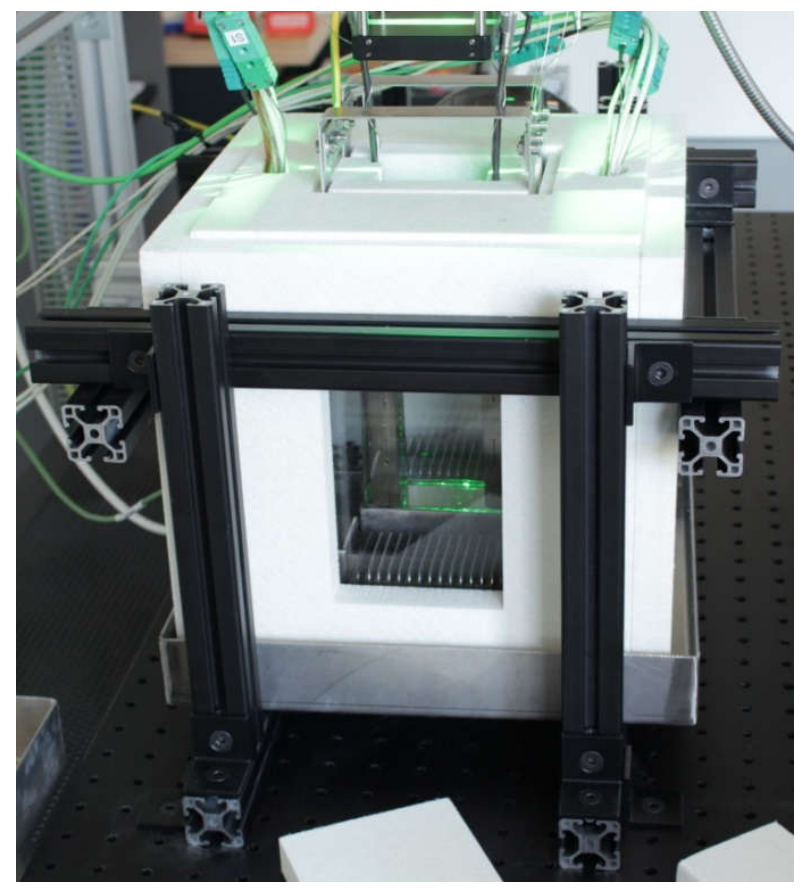

b) Photography of test bench implementation

Figure 2: Test chamber design with inner transparent enclosure that is placed in an outer chamber to reduce heat losses and actively control the surrounding air temperature to achieve near-adiabatic boundary conditions.

To achieve near-adiabatic boundary conditions, the air temperature in the outer chamber is controlled to the same temperature as the PCM. Temperatures in the PCM at the enclosure boundaries are measured with T-type thermocouples of $0.35 \mathrm{~mm}$ and $0.5 \mathrm{~mm}$ diameter, and in the air at the enclosure boundaries with Thermocouples of $0.7 \mathrm{~mm}$ diameter. The thermocouples are placed at six different positions, which are shown in Figure 1. The temperatures of the PCM at the upper side of the enclosure $T_{\mathrm{PCM} \text {,upper side }}$ and at the lower side $T_{\mathrm{PCM}, \text { lower side }}$ are used as set points for three independent trace heater control loops. A first loop controls the air temperature at the bottom boundary below the enclosure $T_{\text {air,below }}$ with a temperature controlled coil heater in a steel plate that is inserted below the PCM enclosure with an air gap in between. The second and the third loop control the temperatures of the air at the side boundaries of the enclosure $T_{\text {air,upper side }}$ and

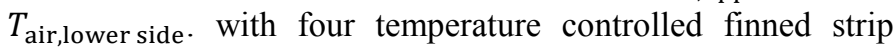
heaters. Two of the finned strip heaters are mounted at the bottom (visible in Figure 2) and two at the top of the outer chamber. This way, thermal stratification in the air can be controlled to be similar as in the PCM, which leads to low heat exchange with the PCM enclosure. The temperature at the top boundary $T_{\text {air,above }}$ is not actively controlled, because heat conduction through the air layer at this boundary is significantly smaller than at the other boundaries and it is not possible to optimally control all the boundary temperatures.

The power of all heaters is controlled with five separate PID loops implemented in LabVIEW ${ }^{\circledR}$ and ran on a compact RIO platform with a Thermocouple module and a digital output module. The controller outputs are transformed into pulse width modulated (PWM) signals that run the heaters with solid states relays. For each of the three air heaters, two cascaded PID loops are used to handle the large reaction time of air heating. The set point of an outer controller is the PCM temperature at an enclosure boundary, e.g. $T_{\mathrm{PCM} \text {,lower side, and }}$ the plant value is the air temperature at the same boundary, e.g. $T_{\text {air,below }}$. The output of the outer controller and the setpoint for the inner controller is the desired temperature of the heater. The plant value of the inner controller is the measured heater temperature and the output is the heater power as PWM signal. This way, a fast reacting inner loop controls the heater temperature and a slow reacting outer loop controls the desired air temperatures at the enclosure boundaries.

The liquid PCM is filled into the enclosure in multiple layers that are solidified subsequently to reduce the formation of air filled cavities. The whole system is then preheated to an initial Temperature that is $1 \mathrm{~K}$ below the melting temperature $T_{\mathrm{m}}$ of the PCM. When temperatures have less than $0.1 \mathrm{~K}$ deviation from the targeted initial temperature $T_{0}$, a steady state is reached and the melting process is started.

\subsection{Material properties}

For these low temperature experiments, we used $n$ octadecane, which melts at a temperature of $28^{\circ} \mathrm{C}$. It is transparent in the liquid state, which allows the use of optical measurement techniques. Its material properties are wellknown. A thorough data set is given by Galione et al. [20], from which the properties provided in Table 1 are adapted. The surface tension was measured by Jasper [21]. 
Table 1: Thermophysical material properties of $n$-octadecane in the solid (s) and liquid (l) state [20,21]. For the liquid state, correlated polynomials are given, where temperatures have to be inserted in K.

\begin{tabular}{ccc}
\hline Property & Unit & Value for $n$-octadecane \\
\hline$\rho_{\mathrm{s}}$ & $\frac{\mathrm{kg}}{\mathrm{m}^{3}}$ & $1010.07-0.80587 \cdot T+0.00012463 \cdot T^{2}$ \\
$\rho_{\mathrm{l}}$ & $\frac{\mathrm{J}}{\mathrm{kg} \mathrm{K}}$ & $2137.1456+2.7186 \cdot(T-273.15)$ \\
\hline$c_{\mathrm{s}}$ & 0.3362 \\
$c_{\mathrm{l}}$ & $\frac{\mathrm{W}}{\mathrm{m} \mathrm{K}}$ & $0.156427-0.0001841779 \cdot(T-273.15)$ \\
\hline$k_{\mathrm{s}}$ & $\log _{10} \mu_{l}=-11.5505+\frac{1670.8}{T}+$ \\
$k_{\mathrm{l}}$ & $0.015675 \cdot T-1.2341 \cdot 10^{-5} \cdot T^{2}$ \\
\hline$\mu_{\mathrm{l}}$ & $\mathrm{Pa} \mathrm{s}$ & $8.9 \cdot 10^{-4}$ \\
\hline$\beta_{\mathrm{l}}$ & $\frac{1}{\mathrm{~K}}$ & 28 \\
\hline$\sigma_{\mathrm{l}}$ & $\frac{\mathrm{N}}{\mathrm{m}}$ & $0.02998-8.428 \cdot 10^{-5} \cdot(T-273.15)$ \\
\hline$T_{\mathrm{m}}$ & ${ }^{\circ} \mathrm{C}$ & 242.454 \\
\hline$L$ & $\frac{\mathrm{kJ}}{\mathrm{kg}}$ &
\end{tabular}

In the solid state, constant properties suffice, because of the low subcooling of $1 \mathrm{~K}$ in this experiment. In the liquid state, however, the property variation over a larger temperature range of $5 \ldots 20 \mathrm{~K}$ is significant, which is why we suggest the correlated polynomials [20].

The thermophysical properties of the steel heaters used to heat up the PCM and the PLEXIGLAS $\AA$ enclosure, in which the PCM is filled, are given in Table 2.

Table 2: Thermophysical properties of enclosure materials.

\begin{tabular}{|c|c|c|c|}
\hline Property & Unit & Steel heaters 1.4301 & PLEXIGLAS ${ }^{\circledR}$ enclosure \\
\hline$\rho$ & $\frac{\mathrm{kg}}{\mathrm{m}^{3}}$ & 7900 & 1180 \\
\hline$c$ & $\frac{\mathrm{J}}{\operatorname{kg~K}}$ & 500 & 1470 \\
\hline$k$ & $\frac{\mathrm{W}}{\mathrm{m} \mathrm{K}}$ & 15 & 0.19 \\
\hline
\end{tabular}

\subsection{Test runs and dimensionless numbers}

Three different experiments with driving temperature differences of 5,10 and $20 \mathrm{~K}$ were conducted. The dimensionless groups are the Rayleigh number $R a_{H}$, the Stefan number Ste, the time dependent Fourier number $F o_{H}(t)$ the Prandtl number $P r$ and the Aspect ratio $A$ :

$$
\begin{aligned}
& R a_{H}=\frac{g \beta \Delta T H^{3}}{v_{1} a_{1}}, \quad \text { Ste }=\frac{c_{1} \Delta T}{L}, \\
& F o_{H}(t)=a_{1} \frac{t}{H^{2}}, \quad \operatorname{Pr}=\frac{v_{1}}{a_{1}}, \quad A=\frac{H}{W} .
\end{aligned}
$$

The driving temperature difference is $\Delta T=\left(T_{\mathrm{w}}-T_{\mathrm{m}}\right)$ between the wall and the melting point of the PCM. There is an ongoing discussion on whether the width or the height should be used as characteristic length. While early research about natural convection in enclosures and many resulting correlations used the width, e.g. Elder [22], theoretical scaling analysis revealed the height as physically meaningful choice [23]. In the end, the characteristic length may be freely chosen, as long as the aspect ratio $A$ of the enclosure is taken into account. In this case, we chose the height $H$ as characteristic length. The aspect ratio in this experiment is $A=4$. The remaining values of the dimensionless numbers are given in Table 3. The material properties are taken at the average liquid temperature $T_{\mathrm{m}}+\Delta T / 2$. The Fourier number is given at the instant of completed melting $\mathrm{Fo}_{H}\left(t_{\mathrm{m}}\right)$.

Table 3: Dimensionless numbers for the three tested temperature differences, i.e. Rayleigh number, Stefan number, Fourier number at time of completed melting and Prandtl number.

\begin{tabular}{cccc}
\hline Dimensionless number & $\Delta T=5 \mathrm{~K}$ & $\Delta T=10 \mathrm{~K}$ & $\Delta T=20 \mathrm{~K}$ \\
\hline$R a_{H}$ & $1 \cdot 10^{8}$ & $2 \cdot 10^{8}$ & $4 \cdot 10^{8}$ \\
Ste & 0.046 & 0.092 & 0.19 \\
$F o_{H}(t)$ & 0.23 & 0.11 & 0.048 \\
$P r$ & 55.1 & 52.2 & 46.5 \\
\hline
\end{tabular}

Depending on the Rayleigh number $R a_{H}$ and the aspect ratio $A$, the heat transfer and flow type in the liquid phase of the PCM can be determined. A criterion for the transition from the pure conduction regime to a convection affected regime was given by Batchelor [24]. We converted the correlation so that the height is used as characteristic length instead of the width and obtained:

$\frac{R a_{H}}{A^{4}} \geq 500$.

This condition is clearly met in all test cases of this study, so that we expect a significant impact of natural convection. The onset of turbulence was studied by Elder [25]. We again converted the correlation so that the height is used as characteristic length. The flow is then characterized to be laminar for

$\mathrm{Ra}_{H}<10^{10}$.

Since all of the experiments performed in this study fulfill this condition, laminar flow was expected.

\section{Measurement techniques}

To measure the relevant quantities of the described validation experiment, multiple measurement techniques were used: the liquid phase fraction of the PCM was measured with shadowgraph imaging, velocities in the liquid phase were measured with particle image velocimetry (PIV) and temperatures were measured with thermocouples. 


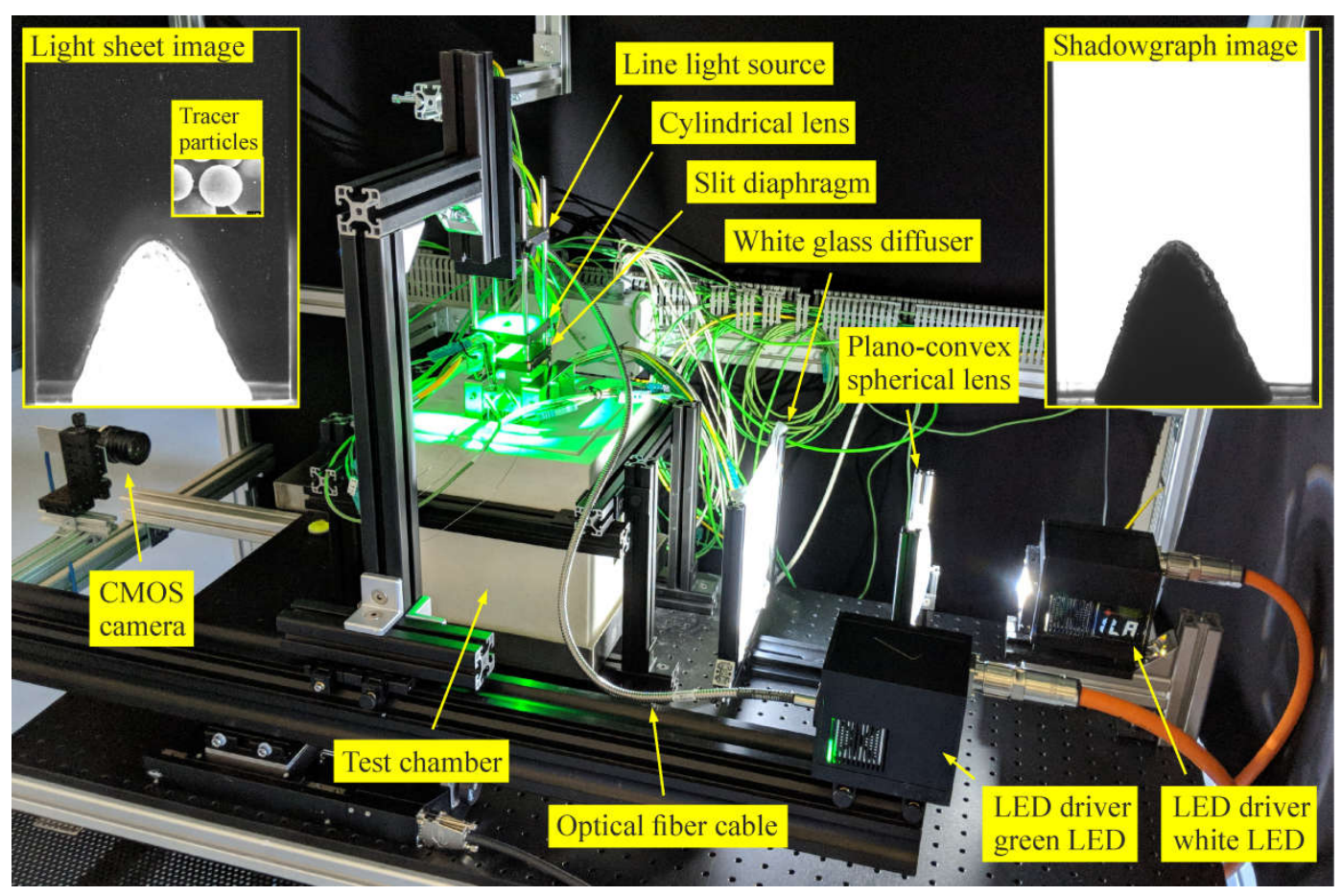

Figure 3: Optical setup for illumination and recording of shadowgraph and light sheet images.

\subsection{Shadowgraph imaging}

To get high-resolution data of the liquid phase fraction, shadowgraph images were digitally recorded and evaluated. As the phase state changes slowly over time, it was sufficient to take a shadowgraph measurement every $60 \mathrm{~s}$ during the experiment. The optical setup that was used for both the shadowgraph and the light sheet images (for PIV measurements) is illustrated in Figure 3.

The test chamber was illuminated from one side with white light emitted from a high power LED CBT-140 by Luminus Devices. The LED was driven by an LED pulsing system (LPS) from Intelligent Laser Applications (ILA) GmbH. The diverging light emitted from the LED was condensed to nearly parallel light with a plano-convex spherical lens with $150 \mathrm{~mm}$ diameter and $300 \mathrm{~mm}$ focal length. To get a more uniform lighting, a white square diffusor plate with $200 \mathrm{~mm}$ edge length was placed in the optical path near to the test section.

\subsection{Particle image velocimetry}

We measured velocities with high resolution in a twodimensional plane using a PIV measurement technique. Fluid flow in the liquid phase of the PCM was visualized with tracer particles. We successfully tested uncoated hollow glass spheres with a mean diameter of $10 \mu \mathrm{m}$ and a density of $1100 \mathrm{~kg} / \mathrm{m}^{3}$ provided by TSI inc. in $n$-octadecane. Although the particle density does not fully match the density of the PCM, the diameter is small enough to ensure good tracing behavior. The sinking (rising) velocity $U_{\mathrm{g}}$ of a particle with diameter $d_{\mathrm{p}}$ and density $\rho_{\mathrm{p}}$ in a quiescent fluid with density $\rho_{\mathrm{l}}$ and viscosity $\mu_{\mathrm{l}}$ due to gravity can be approximately calculated from the Stokes equation, as stated by Raffel et al. [26]:
On the other side of the test section, an industrial monochrome camera Blackfly USB3 by Point Grey recorded the shadowgraph. It features a 1/1.2" CMOS sensor Sony IMX249 with a resolution of $1200 \times 1920$ pixels at a maximum of 41 frames per second. A KOWA lens LM50HC with $50 \mathrm{~mm}$ focal length was used to project the image on the sensor. With this sensor and lens and a working distance of about $500 \mathrm{~mm}$ we obtained the required field of view of $52 \mathrm{~mm} \times 107 \mathrm{~mm}$ slightly larger than the test section dimensions.

The image was read and cropped to the test section region by an acquisition program written in LabVIEW ${ }^{\circledR}$. The conversion factor from image data to the physical space was determined to be $17.4 \mathrm{pixel} / \mathrm{mm}$ with the help of a calibration target. The liquid phase fraction at a certain time was calculated by summing up all of the pixel values in the backlight image, see Figure 4 a), and dividing the result by its maximum possible value, which occurred at the end of the experiment when all PCM was melted.

$$
U_{\mathrm{g}}=\frac{d_{\mathrm{p}}^{2} g\left(\rho_{\mathrm{p}}-\rho_{\mathrm{l}}\right)}{18 \mu_{\mathrm{l}}} .
$$

This equation is valid for a small particle Reynolds number $R e_{\mathrm{p}}=\rho_{\mathrm{l}} U_{\mathrm{f}} d_{\mathrm{p}} / \mu_{1} \ll 1$, which was below $10^{-2}$ in our case. The uncoated hollow glass spheres sank in $n$-octadecane with a velocity of $5 \cdot 10^{-3} \mathrm{~mm} / \mathrm{s}$. This value is sufficiently small in comparison to the maximum fluid velocity of $3 \mathrm{~mm} / \mathrm{s}$, which indicated good tracing ability. To obtain good dynamic tracing behavior, the Stokes number should be small, $S t=\tau_{\mathrm{p}} / \tau_{\mathrm{f}} \ll 1$. We calculated the particle response time $\tau_{\mathrm{p}}$ according to Raffel et al. [26] to $2 \cdot 10^{-6} \mathrm{~s}$ and estimated the characteristic time of our flow problem $\tau_{\mathrm{f}}$ to $1 \mathrm{~s}$. This results in a very low Stokes number of $2 \cdot 10^{-6}$, which underlines the good tracing ability of the particles. 
To illuminate the tracer particles in the $x-y$-plane, see Figure 1, a light sheet was formed. Green light with $525 \mathrm{~nm}$ wavelength was emitted by a high power LED PT-121 from Luminus devices that was mounted in a second LED driver of the LPS system. To form a light sheet, the light was coupled into an optical fiber cable connected to a line light source. A cylindrical lens with $40 \mathrm{~mm}$ height and $50 \mathrm{~mm}$ focal length focused the diverging light from the line light source onto a slit diaphragm with $50 \mathrm{~mm}$ length and $1 \mathrm{~mm}$ width. This way, most of the diffused light due to incoherence, light source size and optical aberrations was cut off. A sharp light sheet was formed that illuminates a plane with 2 to $3 \mathrm{~mm}$ thickness over the whole height of the test section. The light sheet can be offset along the z-axis, see Figure 1, with a linear translation stage to measure in various planes parallel to the $x-y$-plane. The light was reflected by the tracer particles onto the same CMOS camera used for shadowgraphy. However, the two different light sources could not be used at the same time, since the shadowgraph illumination would have outshined the little light reflected from the particles. The other way around, the green light sheet would have illuminated the solid phase inhibiting proper shadowgraph images. The LabVIEW® program automatically controlled alternate shadowgraph and lightsheet illumination, camera configuration, image acquisition and data storage.

A PIV measurement was taken every $60 \mathrm{~s}$ directly after the shadowgraph measurement. At every measurement, a series of 40 images was recorded over $10 \mathrm{~s}$ to obtain transient and average data. A camera frame rate of 4 frames per second ( $250 \mathrm{~ms}$ exposure distance) was chosen to measure velocities, which were below $3 \mathrm{~mm} / \mathrm{s}$. The frame rate was adjusted to allow a maximum particle travel of one fourth within a PIV evaluation window between two successive frames. This has been often stated as optimum value [26]. An exposure time of $30 \mathrm{~ms}$ was just long enough for sufficient light intensity and short enough to avoid motion blur of the particles. However, the camera sensor had to be driven with nearly maximum gain.

The evaluation of velocities was done with the software PIVview conceived by $\mathrm{C}$. Willert, PIVtec GmbH. The underlying algorithms were described by Raffel et al. [26]. The software also provided algorithms for preprocessing, such as image filters, background removal and masking, and for postprocessing, such as calibration and data validation.

Regions that were not to be evaluated, such as the solid phase of the PCM, had to be masked to get proper results from the PIV evaluation. This has usually been done manually. However, in the case of PCM melting, a manual masking of every measurement would have been inefficient. Instead, the shadowgraph images, which were taken before every series of light sheet images, were used to automatically create masks. In Figure 4 a) an original shadowgraph image is shown. Several algorithms from the LABVIEW ${ }^{\circledR}$ Vision Development module, e.g. black and white conversion or closing of holes were used to gain suitable mask images, see Figure $4 \mathrm{~b}$ ). The software PIVview was operated from a windows command prompt, which made it directly accessible from LabVIEW® so that mask images could be assigned to PIV image pairs automatically.
To enhance the contrast and remove unwanted features in the images, a background removal was performed. As background image, the average of the 40 successive light sheet images recorded over $10 \mathrm{~s}$ was used, as shown in Figure $4 \mathrm{c}$ ). Finally, the light sheet images were filtered with a high pass filter with Gaussian weighing and a kernel size of 1.5 pixels. The final preprocessed light sheet image is shown in Figure $4 \mathrm{~d})$.

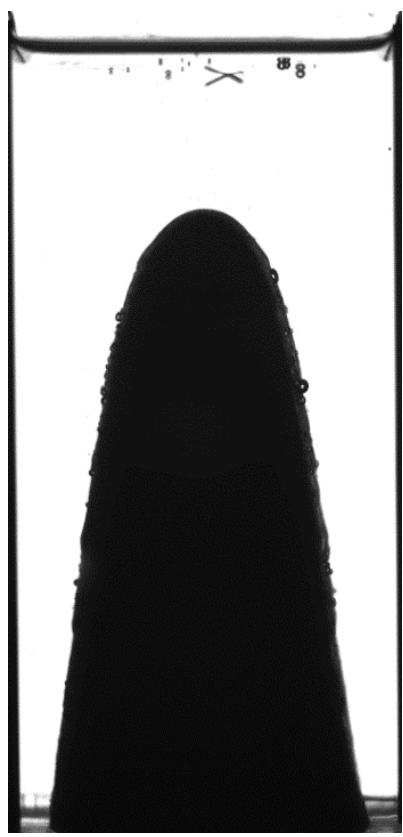

a) Shadowgraph image

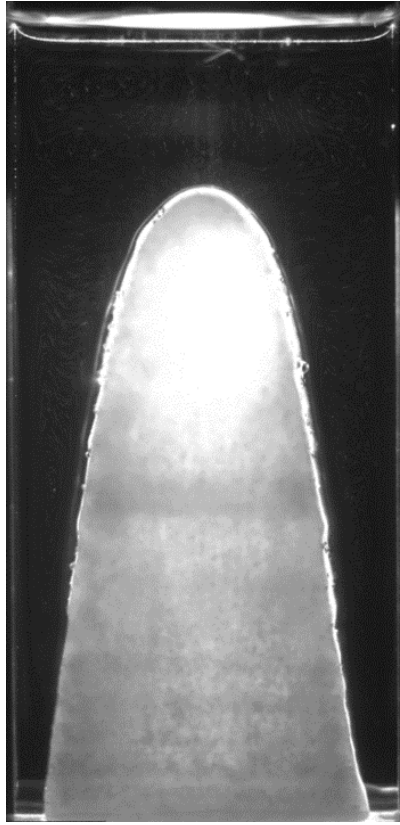

c) Average of 40 light sheet images for background removal

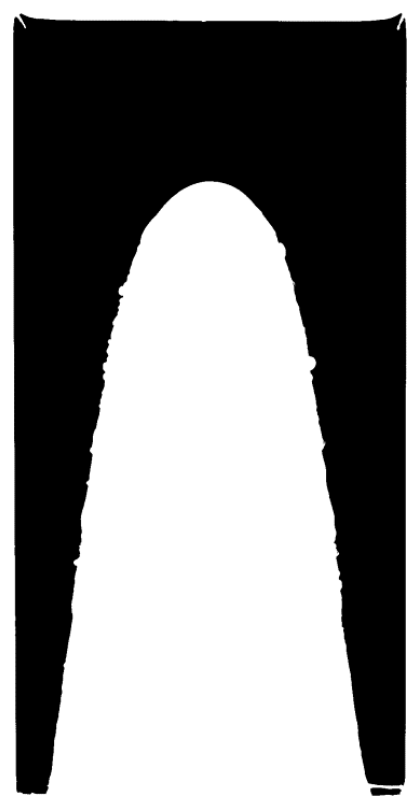

b) Derived mask image

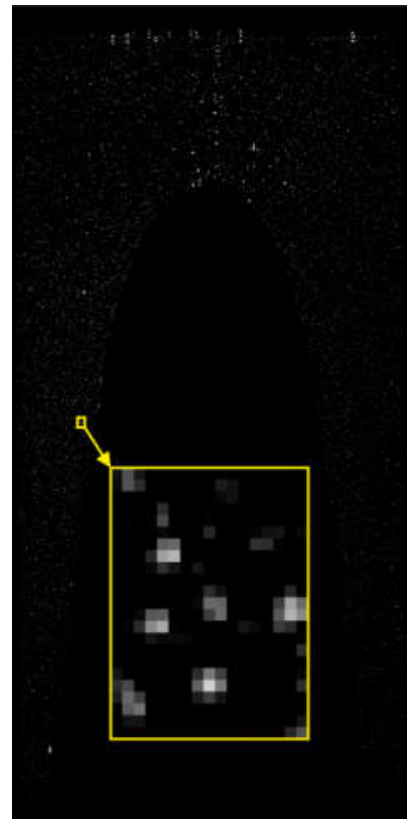

d) Light sheet image after background removal and preprocessing
Figure 4: Mask image creation and PIV preprocessing. 
For PIV evaluation, we selected evaluation windows of 12 pixels in $x$-direction and 48 pixels in $y$-direction with a step size of 8 pixels in $x$-direction and 48 pixels in $y$-direction. This leaded to a vector grid with 112 vectors in $x$-direction and 39 vectors in $y$-direction. The larger window and step size in $y$ direction accounted for higher velocities in this direction and better visualized the resulting larger vectors. In contrast, the smaller window and step size in $\mathrm{x}$-direction accounted for smaller velocities in this direction and better resolved the boundary layers at the heated vertical walls and the phase front of the PCM.

An FFT correlation with multiple repeated correlations (two) and multiplication of correlation planes was used. A multiple-pass interrogation method with 3 passes and sub-pixel image shifting with B-spline interpolation of third order and least squares Gauss sub-pixel peak fit was done.

Finally, for validation of the resulting velocity vectors, a normalized median test and a signal-to-noise ratio test were performed to detect outliers. The detected outliers were either replaced with vectors calculated from the second largest correlation peak or interpolated from neighboring valid vectors.

A last measure to increase reliability and accuracy of the velocity data was to evaluate all the 39 image pairs out of the 40 recorded images over $10 \mathrm{~s}$ and then calculate the average velocity. This filters remaining outliers or false measurements and does not significantly change the solution, since the flow field is quasi-stationary.

\subsection{Thermocouple measurements}

Temperatures during the melting process were measured with K-type thermocouples of $0.35 \mathrm{~mm}$ diameter at three distinct points $T_{\mathrm{PCM}, 1}, T_{\mathrm{PCM}, 2}$ and $T_{\mathrm{PCM}, 3}$ in the test volume, as illustrated in Figure 1. The thermocouples pass through the heating plates from the back side and lie horizontally in the PCM. The positions of the tips of the thermocouples, where the temperatures are measured, are given in Table 4 with respect to the coordinate system shown in Figure 1.

Table 4: Measurement positions of thermocouples in the PCM used to measure temperatures during the melting process.

\begin{tabular}{lccc}
\hline Thermocouple & PCM,1 & PCM,2 & PCM,3 \\
$\begin{array}{l}\text { Measurement position } \\
(x, y, z) / \mathrm{mm}\end{array}$ & $(5,90,25)$ & $(15,50,25)$ & $(25,10,25)$ \\
\hline
\end{tabular}

\section{Results and Discussion}

We successfully conducted and evaluated several test runs with the previously presented validation experiment using the described measurement techniques. We tested three different driving temperature differences $\Delta T$ of 5,10 and $20 \mathrm{~K}$, for which we already provided the corresponding dimensionless numbers in section 3.4. We focused on the experiment with $\Delta T=10 \mathrm{~K}$, for which we present a visualization of the melting process showing the phase state and velocity vectors in section 5.1. We repeated this experiment four times as similar as possible to check reproducibility and to calculate statistical errors. After performing an error assessment in section 0 and analyzing the actually measured boundary temperatures in section 5.3, we present the quantitative results of liquid phase fractions, velocity extrema and temperatures in section 5.4. Finally, we show results of the liquid phase fraction in dimensionless form and derive a suitable correlation in section 5.5 .

\subsection{Visualization of the transient melting process}

The transient melting process is visualized in Figure 5 after $1800 \mathrm{~s}$ and $3600 \mathrm{~s}$ showing the average of 40 light sheet images taken over $10 \mathrm{~s}$ in the background. The solid phase of the PCM appears in white and light grey shades and the liquid phase in black. On top of that, the evaluated velocities are shown with vectors colored by velocity magnitude. In Figure 6, the melting process is visualized after $5400 \mathrm{~s}$ and $7200 \mathrm{~s}$. Velocities are presented again with vectors colored by velocity magnitude, but shadowgraph images are shown in the background, where the solid phase appears in black and the liquid phase in white.

We observe that melting is dominated by natural convection due to the typical melting not only from left to right but also from top to bottom due to thermal stratification, which leads to a curved phase front shape. We can clearly observe the boundary layer of the upward flow at the heated walls and the boundary layer of the inclined downward flow at the cold solid phase. We can also notice larger maximum velocity magnitudes in the downward flow at the cold solid phase as in the upward flow at the heated plates.

Furthermore, we can see larger maximum velocity magnitudes at the left wall than at the right wall. At first sight, this is unexpected regarding the symmetry of the problem. But in an experiment we would not expect a perfect symmetry, either. We assume that the temperature of the heated wall on the left is higher than that on the right side. Although both plates were controlled to the same temperature, measurement uncertainties could allow slightly different temperatures. We conclude that already a small temperature difference may lead to a visible asymmetry in the flow field. Nevertheless, the phase front remains symmetric and therefore we also conclude that the flow field asymmetry has only a minor impact on the melting process.

The filling of the PCM in many layers with subsequent solidification reduced the amount of cavities in the solid, but it could not prevent them completely. We can still see gas bubbles originating from the solid material and rising to the top surface. Fortunately, the rising bubbles do not significantly decrease the PIV measurement accuracy, because their velocities are out of the range captured by PIV evaluation. However, the rising gas bubbles entrain fluid along and could thus affect the ideally stated melting problem. To completely prevent cavities in the solid, the PCM would have to be solidified in a vacuum environment, so that no air can diffuse into the cavities formed by shrinking of the solid with solidification. Nevertheless, the formation of shrinkage cavities is a natural phenomenon that also occurs in real latent heat storage systems. 


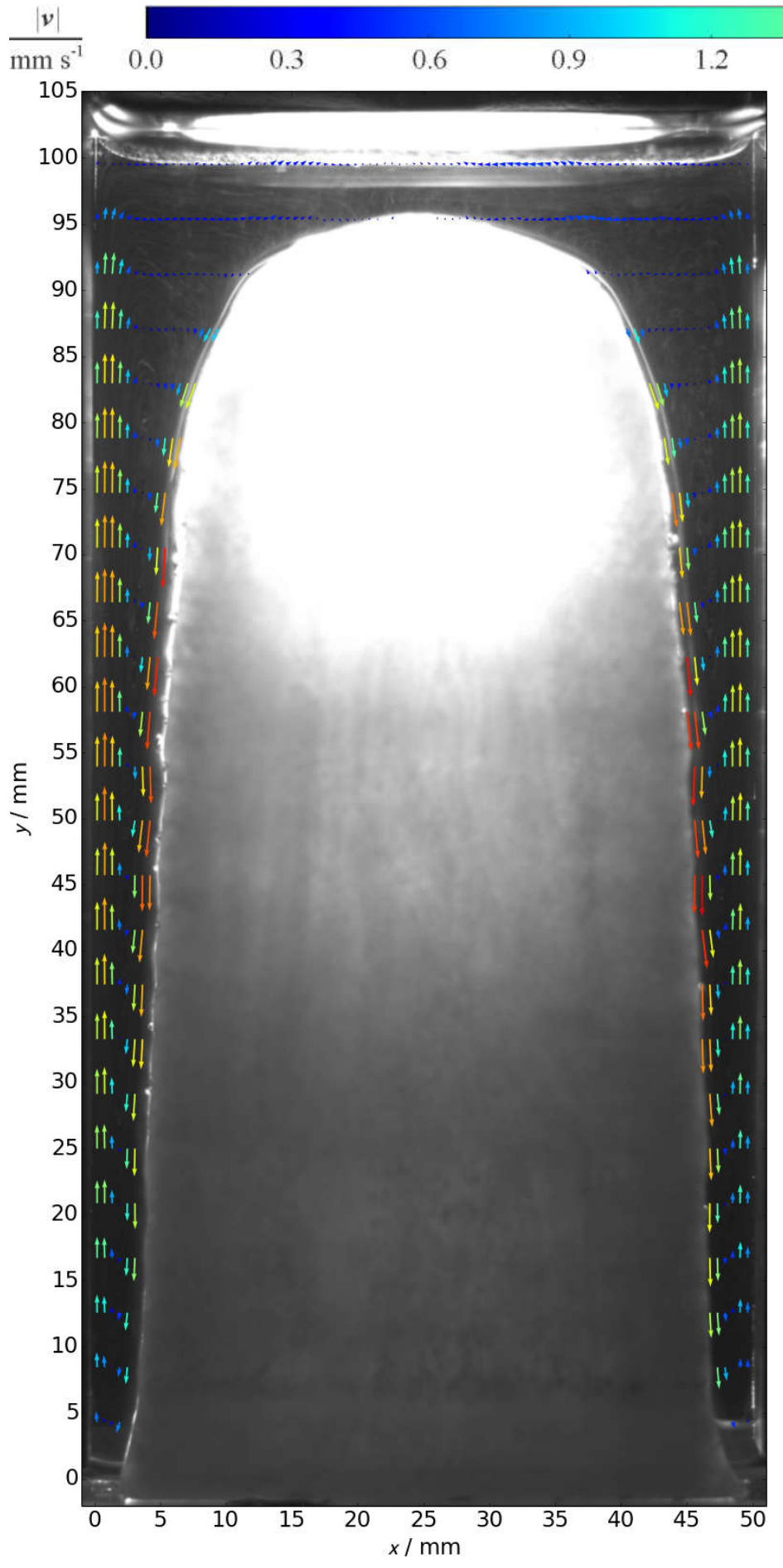

a) $1800 \mathrm{~s}$

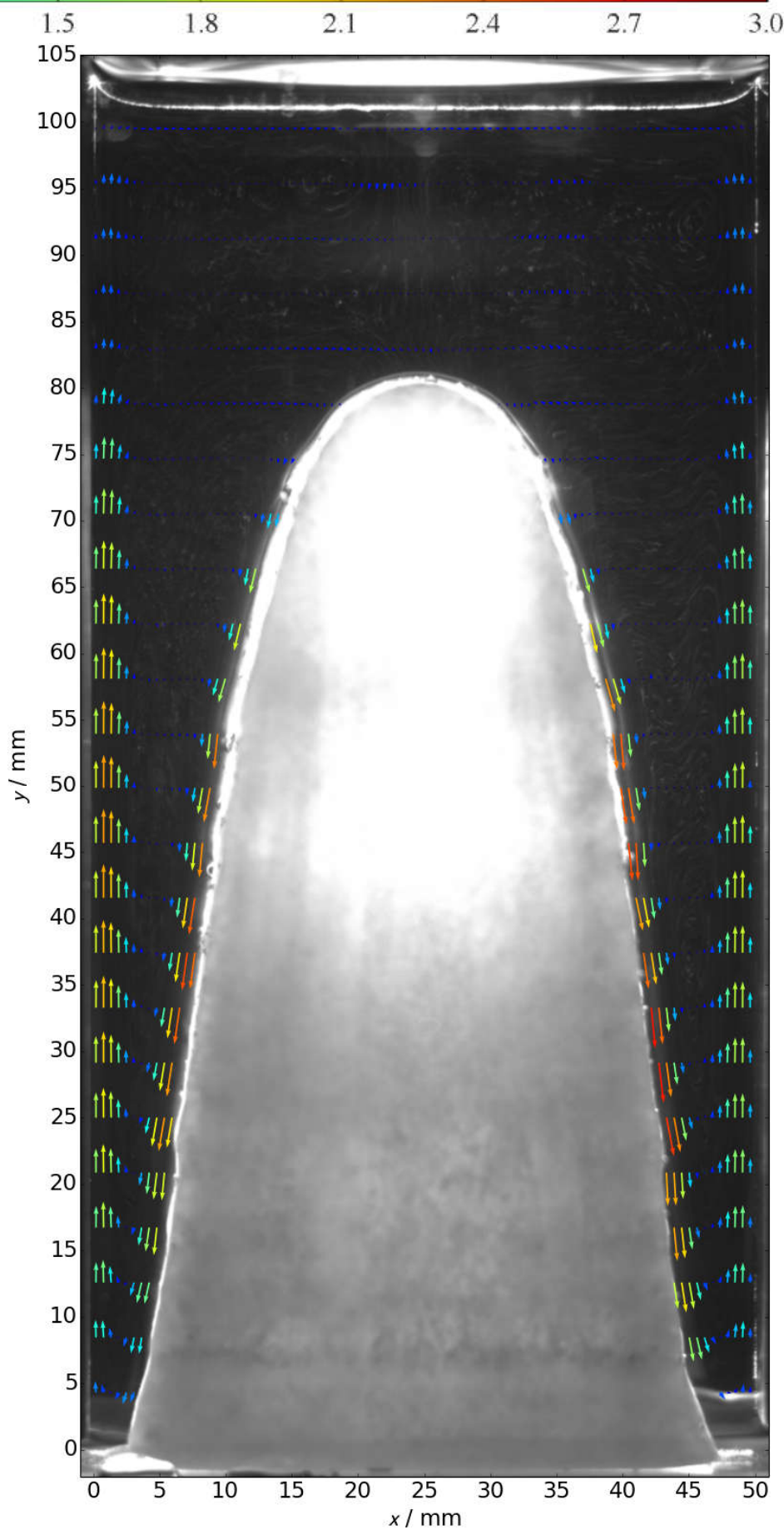

b) $3600 \mathrm{~s}$

Figure 5: Transient visualization of phase state by averaged light sheet images and velocities by vectors colored by magnitude. 


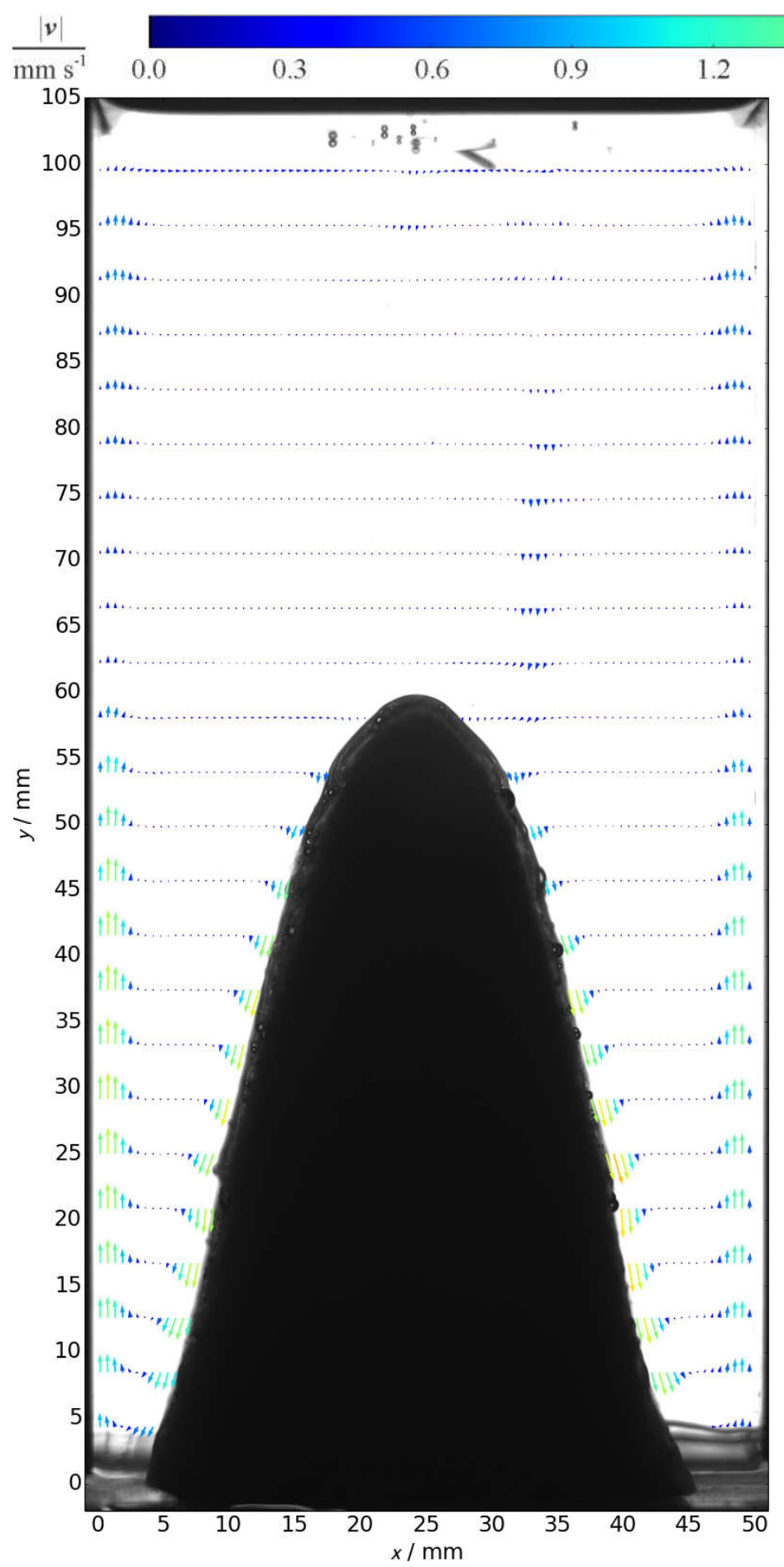

a) $5400 \mathrm{~s}$

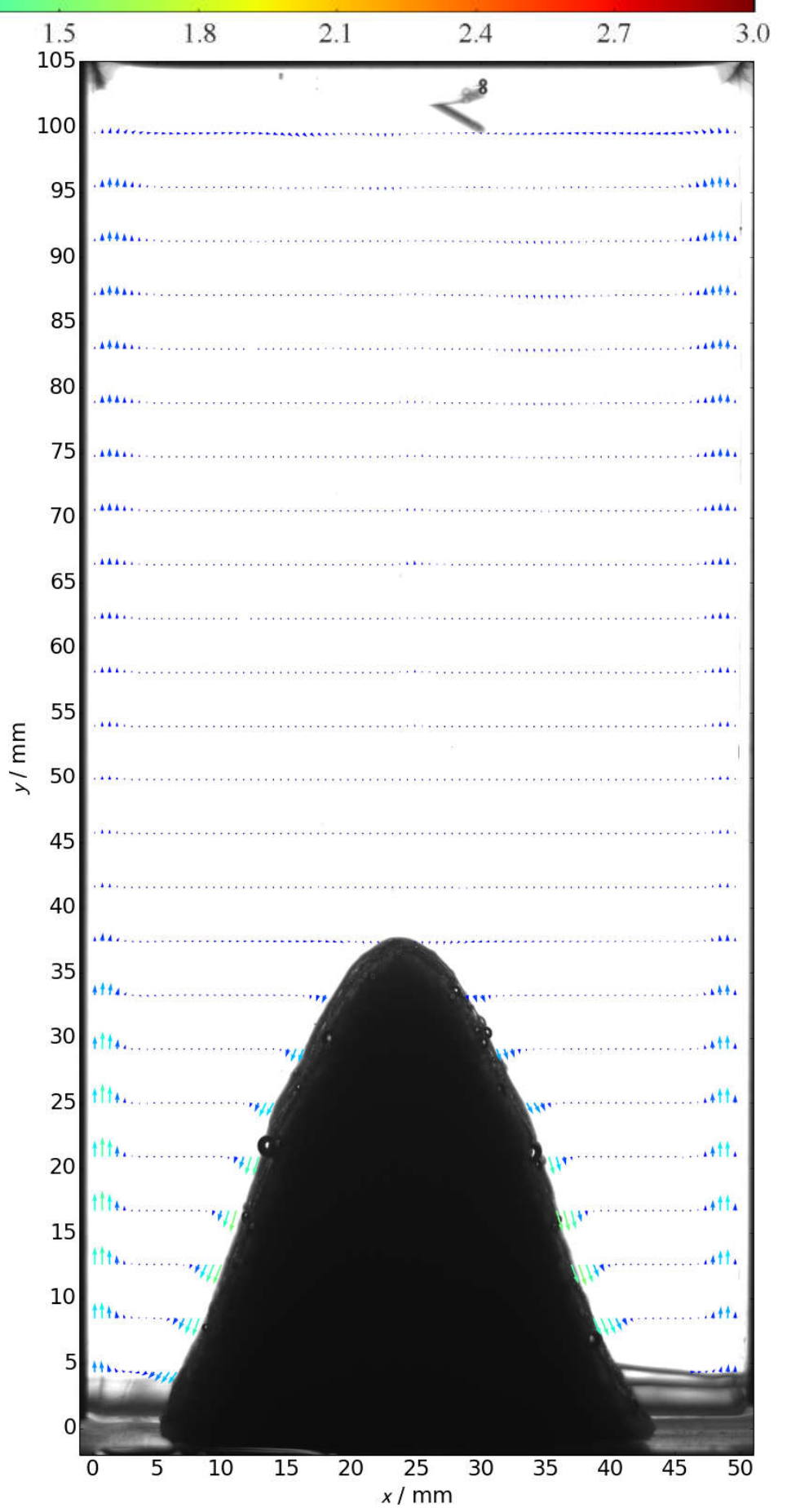

b) $7200 \mathrm{~s}$

Figure 6: Transient visualization of phase state by back light images and velocities by vectors colored by magnitude. 
We conclude that the high resolution in velocity from PIV measurements visualizes the whole flow field and gives detailed insight into the phenomenon of natural convection during melting of a PCM.

\subsection{Measurement and evaluation errors}

The estimation of systematic and statistical measurement errors is crucial to judge measurement accuracy. We analyzed the measurement of the phase state by shadowgraphy, the measurement of velocity by PIV and the measurement of temperatures by thermocouples. We aimed to include the most important systematic error sources and repeated measurements of the same experiment four times to gain statistical errors.

\subsubsection{Liquid phase fraction measurement}

The liquid phase fraction, which is a dimensionless value between 0 and 1 , was obtained from the shadowgraph images, as explained in section 4.1. The systematic errors were mainly due to optical distortions, perspective and obstacles in the optical path, e.g. thermocouples. We assumed that these errors should be well below two percent, which leaded to an absolute error range of \pm 0.02 .

The statistical analysis of four repeated measurements of the liquid phase fraction over $11700 \mathrm{~s}$ at 19 time steps with a step size of $600 \mathrm{~s}$ revealed $95 \%$ confidence intervals between \pm 0.0066 and \pm 0.034 . Conclusively, the total systematic and statistical errors were between \pm 0.027 and \pm 0.054 .

\subsubsection{Velocity measurement}

The velocity was measured by optical imaging of particle movement illuminated by a light sheet and optical imaging to a camera sensor. The data was evaluated by a PIV software. We identified possible systematic measurement and evaluation errors due to 1) distortions in the optical imaging and calibration procedure from image to physical space, 2) long exposure motion blur of particle images, 3) particle inertia or sinking of particles due to density mismatch with the fluid and 4) the PIV evaluation algorithms.

As stated by Adrian and Westerweel [27], distortion errors are often negligibly small. Since we don't have curved surfaces in our optical path and the focal length of our camera lens was not exceptionally wide, we neglected distortion errors. The calibration from the image to physical space was done by measuring the known physical distance of $100 \mathrm{~mm}$ on a calibration target in pixel values. Assuming that we measured with a precision of 10 pixels, we had a total error of 0.1 $\mathrm{pixel} / \mathrm{mm}$ in the conversion factor. This induced an error of $\pm 1.7 \cdot 10^{-2} \mathrm{~mm} / \mathrm{s}$ on the maximum velocity with magnitude $3 \mathrm{~mm} / \mathrm{s}$.

We selected the exposure time of the light source in that manner that a particle only traveled a maximum distance of one pixel during exposure to avoid excessive motion blur. Nevertheless, we estimated the resulting error to $\pm 0.1 \mathrm{~mm} / \mathrm{s}$.

As described in section 4.2, the Stokes number for the tracer particles in the fluid was $2 \cdot 10^{-6}$, which indicated negligible dynamic tracing error. The sinking velocity of
$-5 \cdot 10^{-3} \mathrm{~mm} / \mathrm{s}$ was also small, but we still included this value in our systematic error.

As stated by Adrian and Westerweel [27], the PIV evaluation error is mostly between 0.1 and 0.2 pixels. We assumed a value of 0.2 pixels. However, due to averaging of velocities from 39 evaluations of 40 subsequent images, this value reduced to 0.03 pixels. Converted to physical space, this corresponded to a velocity error of $\pm 7 \cdot 10^{-3} \mathrm{~mm} / \mathrm{s}$.

The sum of the systematic errors we found was $\pm 0.15 \mathrm{~mm} / \mathrm{s}$. The statistical analysis of four repeated measurements of the maximum and minimum velocities in $x$ and $y$-direction over $11400 \mathrm{~s}$ at 19 time steps with a step size of $600 \mathrm{~s}$ revealed $95 \%$ confidence intervals between $\pm 0.02 \mathrm{~mm} / \mathrm{s}$ and $\pm 0.2 \mathrm{~mm} / \mathrm{s}$ in $x$-direction and between $\pm 0.05 \mathrm{~mm} / \mathrm{s}$ and $\pm 0.7 \mathrm{~mm} / \mathrm{s}$ in $y$-direction. The resulting sums of systematic and statistical errors lay between $\pm 0.2 \mathrm{~mm} / \mathrm{s}$ and $\pm 0.4 \mathrm{~mm} / \mathrm{s}$ in $x$-direction and between $\pm 0.2 \mathrm{~mm} / \mathrm{s}$ and $\pm 0.5 \mathrm{~mm} / \mathrm{s}$ in $y$-direction.

\subsubsection{Temperature measurement}

The thermocouples of type $\mathrm{K}$ used for measuring temperatures had a specified absolute accuracy of $\pm 1.5 \mathrm{~K}$. However, the phase change temperature of three different materials was measured with multiple thermocouples with a deviation below $0.5 \mathrm{~K}$. Furthermore, temperature measurements from all the installed thermocouples lay within $0.1 \mathrm{~K}$ in thermal equilibrium. Thus, we concluded that the systematic measurement error was at most $\pm 0.5 \mathrm{~K}$.

The statistical analysis of three repeated measurements of temperatures at 40 time steps over $12000 \mathrm{~s}$ with a step of $300 \mathrm{~s}$ revealed $95 \%$ confidence intervals between $\pm 0.005 \mathrm{~K}$ and $\pm 6.6 \mathrm{~K}$. The larger confidence intervals only occurred over a short period of time during melting in the vicinity of the thermocouple, which resulted in a large temperature gradient and therefore a large variation between measurements. Most of the time, the confidence interval was much smaller. The resulting sums of systematic and statistical errors lay between $\pm 0.5 \mathrm{~K}$ and $\pm 7.1 \mathrm{~K}$.

\subsection{Measured boundary conditions}

In the test case definition in section 3.1, the ideal boundary conditions were specified. The initial temperature was $T_{0}=$ $27{ }^{\circ} \mathrm{C}$ and the wall temperature was raised to $T_{\mathrm{w}}=38^{\circ} \mathrm{C}$ at time $t_{0}=0$. All non-heated walls would have to be adiabatic in the ideal case. However, these ideal conditions cannot completely be realized in an experiment. To analyze the experimental boundary conditions, we measured temperatures at the different boundaries during the melting experiment and roughly estimated heat flow rates.

\subsubsection{Boundary temperatures}

The wall temperatures at three different heights of a heating plate measured during a melting experiment are shown in Figure 7. The measurement positions can be found in Figure 1. Every plotted value is a mean value from four different measurements of the same experiment. The temperature in the 
middle was used for PID-control and therefore settled at the designated temperature of $38^{\circ} \mathrm{C}$ after a short overshoot. The time average of this middle temperature was also about $38^{\circ} \mathrm{C}$.

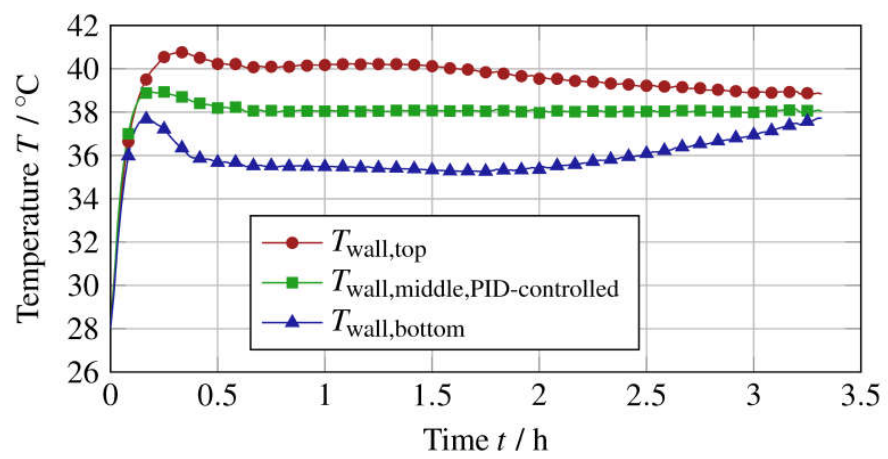

Figure 7: Temperatures measured during a melting experiment at one of the heated walls at the top, middle and bottom. The temperature at the middle location was used for PID-control of the wall temperature.

The thermal stratification in the PCM due to natural convection and the finite thermal conductivity of the stainless steel heating plates, which is still about 100 times higher than that of the liquid PCM, lead to a temperature variation over the height of the heated wall. The variation over time mainly depends on the heat transfer rate from the heater into the PCM. During the melting process with strong natural convection, the heating plates cannot compensate the thermal stratification in the PCM. Only in the pure conduction regime at the very beginning and after completed melting at the end the heating plates have a uniform temperature over the height. The time average of the top temperature is $39.5^{\circ} \mathrm{C}$ and that of the bottom temperature is $35.9{ }^{\circ} \mathrm{C}$.

This temperature variation over the height is clearly a deviation of the experiment from the originally stated ideal case. But the impact on the results has yet to be analyzed, e.g. in a numerical comparison study of ideal and real wall boundary conditions. Nevertheless, the average of the mean temperatures at the three height levels is $37.9^{\circ} \mathrm{C}$, which is close to the designated $38{ }^{\circ} \mathrm{C}$ and thereby ensures the ideal wall temperature in a mean sense.

The temperatures measured at six locations of the remaining non-heated walls are shown in Figure 8; for the locations refer to Figure 1. At one of the side walls, temperatures were measured at an upper and a lower location both in the PCM at the inside of the enclosure and in the air at the outside of the enclosure. Additionally, the air temperature was measured above the enclosure at the lid window and directly below the enclosure.

At the upper position of the enclosure side wall, the temperature in the air is lower than that in the PCM. At the lower position of the enclosure side wall, the temperature in the air is higher than that in the PCM. This is due to the control algorithm which controls both air temperatures to both PCM temperatures in a mean sense. Since the air has different properties than the PCM, the thermal stratification is different in both materials and a better agreement is not achievable. The air temperature above the enclosure was not actively controlled and is just a result of this control strategy. It is lower than the air temperature at the side, because the enclosure side walls are also heated by the main heaters and, as a result, the air heaters lower their power to not introduce too much heat, which, together with large heat losses at the top, results in a lower air temperature above the PCM enclosure. The air temperature below the enclosure matches the PCM temperature at the lower position. The air volume below the enclosure can be well controlled, because it is separated and insulated from the rest of the air in the outer enclosure.

Conclusively, we can see that the quasi-adiabatic control strategy leads to low differences between air and PCM temperatures at the enclosure boundaries.

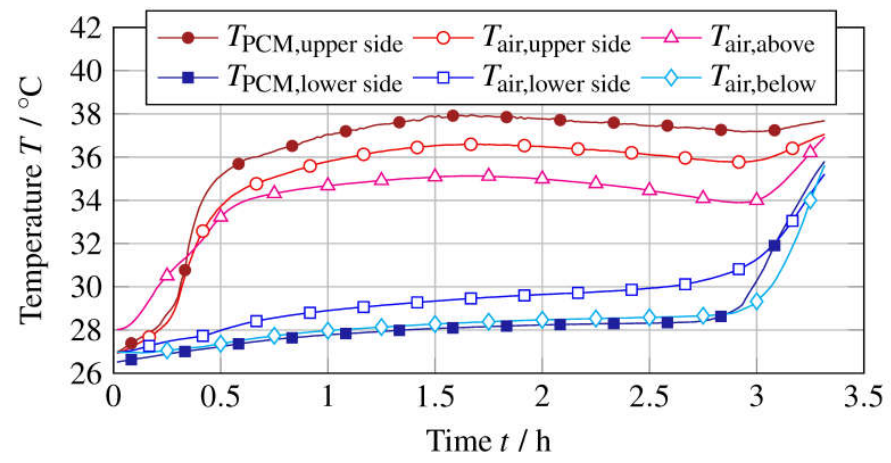

Figure 8: Temperatures measured at six locations of the non-heated walls.

\subsubsection{Boundary heat flow rates}

To estimate the influence of the remaining heat flow over the quasi-adiabatic boundaries on the melting process, we approximated the heat flow rates through the boundaries and compared them to the power of the heating plates that heat up the PCM.

The heat flow rates at the three different boundaries "below", "sides" and "above" were approximately calculated from the difference of the measured temperatures between PCM and air shown in Figure 8. The Fourier law for heat conduction through the enclosure walls was used for the sides and bottom boundaries. The Fourier law with effective thermal conductivity was used to calculate heat conduction or natural convection in the air layer above the PCM. Heat radiation through this air layer was also approximately calculated. We summed up all these boundary heat flow rates to obtain a total boundary heat flow rate. For comparison, the heat flow rate that is transferred by the heating plates into the PCM was approximately calculated by the power rating of the heaters and the duty cycle of the PWM signal.

In total, relative to the heating power, $3.4 \%$ of heat was lost and $1.9 \%$ was gained through the boundaries. This confirms a minor influence of the boundaries on the melting process and with it the effectiveness of our quasi-adiabatic active control approach of boundary temperatures.

\subsection{Quantitative results for validation}

Finally, we present the quantitative results of our experiment for the purpose of numerical model validation. The relevant quantities are the liquid phase fraction, velocities in 
the liquid phase of the $\mathrm{PCM}$ and temperatures at distinct positions in the PCM.

\subsubsection{Liquid phase fractions over time}

The liquid phase fractions were calculated from shadowgraph images taken every $60 \mathrm{~s}$ during the experiment, see section 4.1. The temporal evolutions of liquid phase fractions for three different experiments with driving temperature differences $\Delta T=T_{\mathrm{w}}-T_{\mathrm{m}}$ of 5,10 and $20 \mathrm{~K}$ are plotted in Figure 9.

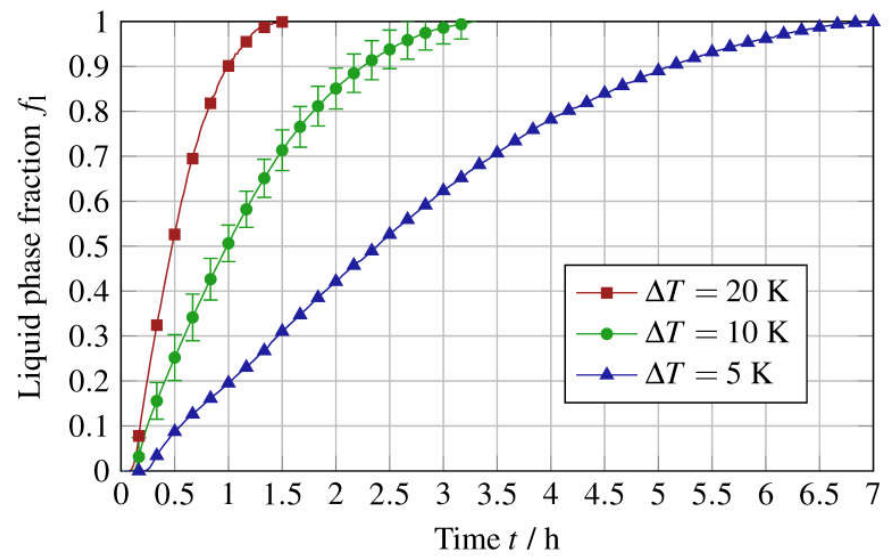

Figure 9: Liquid phase fractions over time of three different experiments with driving temperature differences $\Delta T$ of 5,10 and $20 \mathrm{~K}$.

The melting times are $5460 \mathrm{~s}$ for $\Delta T=20 \mathrm{~K}, 11880 \mathrm{~s}$ for $\Delta T=10 \mathrm{~K}$ and $25560 \mathrm{~s}$ for $\Delta T=5 \mathrm{~K}$. For the experiment with $\Delta T=10 \mathrm{~K}$, error bars are provided. They show the sum of the systematic error and the statistical confidence interval from four repeated measurements at selected times, as calculated in section 5.2.1.

\subsubsection{Velocity extrema over time}

The full velocity field, as illustrated in Figure 5 and Figure 6 , contains a lot of data and is hard to quantify. Therefore, we calculated the maximum and minimum velocities in both the $x$ and $y$-direction. The results are plotted over time in Figure 10. Error bars show the sum of the systematic error and the statistical confidence interval from four repeated measurements at selected times, see also section 5.2.2.

It is clearly seen that vertical velocities in $y$-direction are significantly larger than horizontal velocities in $x$-direction. This is expected, because the buoyancy force acts in $y$ direction at the walls and in negative $y$-direction at the phase boundary. Furthermore, the velocity magnitudes in positive and negative $x$-direction are similar. However, the magnitude in positive $y$-direction is about $20 \%$ lower than in negative direction.

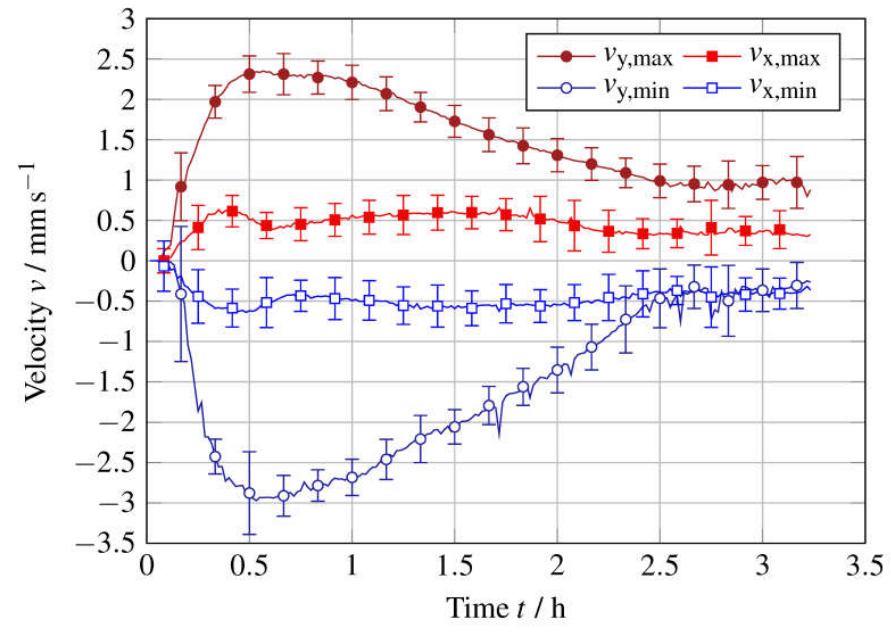

Figure 10: Maximum and minimum velocities in $x$ - and $y$-direction over time of the experiment with $\Delta T=10 \mathrm{~K}$.

\subsubsection{PCM Temperatures}

Temperatures inside the PCM $T_{\mathrm{PCM}, 1}, T_{\mathrm{PCM}, 2}$ and $T_{\mathrm{PCM}, 3}$ at the three distinct measurement positions shown in Figure 1 are plotted in Figure 11. Error bars show the sum of the systematic error and the statistical confidence interval from four repeated measurements at selected times, as calculated in 5.2.3. The error bars are wider at high temperature gradients due to larger deviations of repeated measurements. The time when the melting front reached the thermocouple with rapid increase in temperature could not exactly be reproduced. The predominant remaining time, however, errors were sufficiently small.

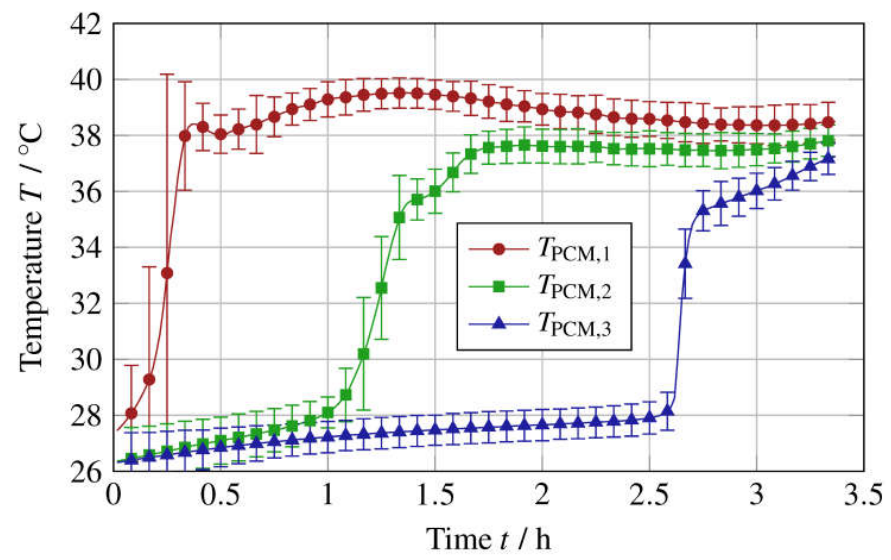

Figure 11: Temperatures in the PCM over time of the experiment with $\Delta T=10 \mathrm{~K}$ at three distinct measurement positions.

We observe that the temperatures show the progression expected from a phase change process. The temperatures only slightly exceeded the targeted temperature of $38^{\circ} \mathrm{C}$, which was due to the slight non-uniformity of the heating plates, compare Figure 7.

\subsection{Scaling of the melting process}

There have been sophisticated theoretical scaling analyses such as by Jany and Bejan [23] that divide the melting process in several regimes and derive the scaling laws theoretically. 
However, a widely used simplified approach is to assume that the melting process is dominated by the convection regime. The state of the art and our scaling procedure are already given by Vogel et al [28], where a scaling of a similar test problem has been derived from a numerical parameter study of rectangular enclosures with varying dimensions. In this work, we extended this study to scale the liquid phase fraction curves of the three experiments with varying driving temperature differences that were shown in Figure 9. We used the dimensionless numbers $F o_{H}(t)$, Ste, $R a_{H}$ and $A$ given in Table 3 . We retained the exponents from the previous study [28], but converted them from the width to the height as characteristic length. Only the exponent of $A$ changes from $-1 / 4$ to $5 / 4$. We additionally used Ste to scale the varying driving temperature difference. We tried to optimize the exponent of Ste, but the best results were obtained with a value close to unity. An additional constant with a value of 0.78 set the maximum of the scaled time to 1 . The resulting scaled time is:

$$
\tilde{t}=0.78 \mathrm{Fo}_{H}(t) \text { Ste } R a_{H}{ }^{\frac{1}{6}} A^{\frac{5}{4}} .
$$

Finally, we fit the error function $b_{1} \cdot \operatorname{erf}\left(b_{2} \cdot \tilde{t}\right)$ to the scaled liquid phase fraction curves. We used a regression analysis that minimizes the sum of all least square residuals to a value of 0.06 with parameters $b_{1}=1.04$ and $b_{2}=1.57 \approx$ $\pi / 2$. This leads to a curve-fit function for the liquid phase fraction over scaled time $\tilde{t}$ :

$$
f_{1}(\tilde{t})=1.04 \cdot \operatorname{erf}\left(\frac{\pi}{2} \cdot \tilde{t}\right) .
$$

The three liquid phase fraction curves over scaled time $\tilde{t}$ and the curve-fit function (6) are plotted in Figure 12. We see that the liquid phase fraction curves coincide due to the scaling and the curve-fit represents the data well.

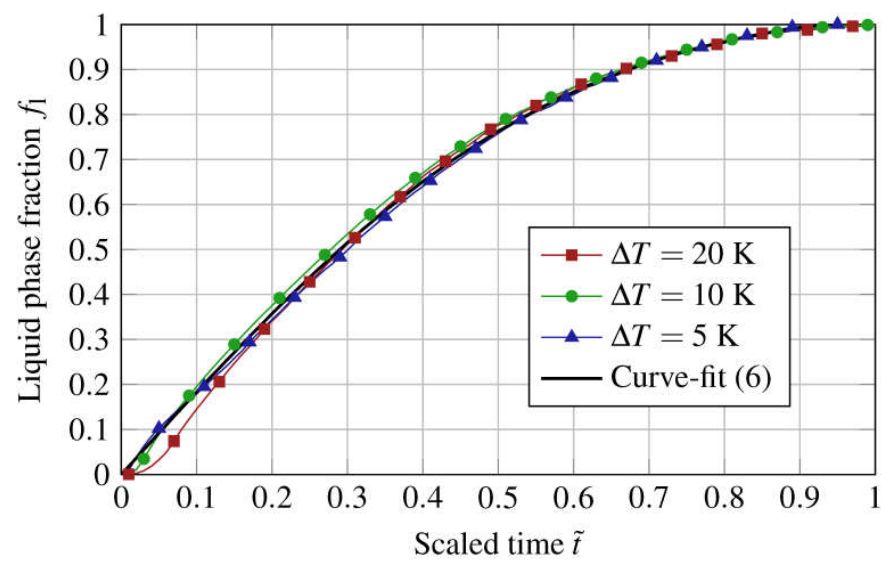

Figure 12: Liquid phase fractions of three different test runs with varying temperature difference $\Delta T$ and the least squares curve-fit correlation (6) plotted over scaled time $\tilde{t}$.

In the previous study by Vogel et al. [28], a scaling for various enclosure dimensions was done. In this study, we expanded the analysis on different driving temperature differences. Combining the two studies, the presented scaling (5) and the curve-fit correlation (6) span a large parameter range including enclosure heights of 25 to $1000 \mathrm{~mm}$, enclosure half widths of 5 to $25 \mathrm{~mm}$, aspect ratios of 0.5 to 40 and driving temperature differences from 5 to $20 \mathrm{~K}$. Restrictions of this correlation are, however, the convection regime bounded by equation (2) and the laminar regime bounded by equation (3).

\section{Conclusions}

A novel test case for the melting of a PCM was defined and a validation experiment was built, run and evaluated. The PCM was contained in a rectangular enclosure and was heated from vertical opposing sides by two heating plates.

We identified three main challenges of such an experiment. The first is that the heating plates may not conduct heat well enough and, due to the thermal stratification in the PCM, establish a temperature variation over the height. The significance of this problem should be investigated in further studies. Since we provided the temperatures at three positions over the height and with time, this boundary condition may be easily implemented in a simulation. The second challenge is a symmetric heating from both sides. We found a larger flow velocity magnitude in one of the two wall boundary layers. Although both plates were controlled to the same temperature, measurement uncertainties could allow slightly different temperatures. We conclude that already a small temperature difference may lead to a visible asymmetry in the flow field. Nevertheless, the phase front remains symmetric and we therefore conclude that the flow field asymmetry has only a minor impact on the melting process. The third challenge is heat flow over the ideally adiabatic boundaries, which can affect the melting process. We minimized the heat flow rates over the boundaries with an active quasi-adiabatic control approach of trace heaters in a surrounding air chamber. We estimated a remaining total heat loss of $3.4 \%$ and heat gain of $1.9 \%$ relative to the heat transferred by the heating plates. We therefore conclude that there was a negligible effect of heat transfer through the boundaries in this experiment.

Our main results are measurements of the phase state and velocities in the liquid phase of the PCM with high spatial and temporal resolution. The optical measurement techniques of shadowgraphy and PIV produced highly resolved data of the phase state and velocity in a two-dimensional vertical plane. With an automated measurement technique, we took approximately 200 measurement sets in every experiment to obtain a temporal resolution of the whole melting process. Each measurement set included a shadowgraph image and a series of 40 lightsheet images over $10 \mathrm{~s}$ to obtain transient and averaged velocity data. The measurement errors of liquid phase fraction, velocities and temperatures are sufficiently low and confirm accurate results. These comprehensive and accurate data can be used for validation of numerical models for phase change processes.

An additional important result is a least-squares curve fitting correlation for the liquid phase fraction in dimensionless form. This correlation can be used to predict similar melting processes in the given parameter range, which is valuable in system design and optimization. 


\section{Acknowledgments}

The authors are grateful to Markus Eck, Stefan Zunft and André Thess for inspiring discussions on validation experiments, Lisa Böhm and Janina Hagedorn for assisting in the test bench set-up during their Bachelor's theses and Vanessa Schönfelder for performing a measurement campaign during her Master's thesis.

\section{References}

[1] IPCC, Climate Change 2014: Synthesis Report. Contribution of Working Groups I, II and III to the Fifth Assessment Report of the Intergovernmental Panel on Climate Change [Core Writing Team, R.K. Pachauri and L.A. Meyer (eds.)], Geneva, Switzerland, 2014.

[2] International Energy Agency (IEA), Technology Roadmap: Energy storage, 2014.

[3] H. Mehling, L.F. Cabeza, Heat and cold storage with PCM, Springer-Verlag Berlin Heidelberg, 2008. doi:10.1007/978-3540-68557-9.

[4] T. Bauer, W.-D. Steinmann, D. Laing, R. Tamme, Thermal Energy Storage Materials and Systems, in: Annu. Rev. Heat Transf., 2012: pp. 131-177. doi:10.1615/AnnualRevHeatTransfer.2012004651.

[5] S. Jegadheeswaran, S.D. Pohekar, T. Kousksou, Exergy based performance evaluation of latent heat thermal storage system: A review, Renew. Sustain. Energy Rev. 14 (2010) 2580-2595. doi:10.1016/j.rser.2010.07.051.

[6] S. Kadri, B. Dhifaoui, Y. Dutil, S. Ben Jabrallah, D.R. Rousse, Large-Scale Experimental Study of a Phase Change Material: Shape Identification for the Solid-Liquid Interface, Int. J. Thermophys. 36 (2015) 2897-2915. doi:10.1007/s10765-015-1935-y.

[7] N.S. Dhaidan, J.M. Khodadadi, Melting and convection of phase change materials in different shape containers: A review, Renew. Sustain. Energy Rev. 43 (2015) 449-477. doi:10.1016/j.rser.2014.11.017.

[8] N. Hale, R. Viskanta, Photographic observation of the solidliquid interface motion during melting of a solid heated from an isothermal vertical wall, Lett. Heat Mass Transf. 5 (1978) 329-337.

[9] C. Benard, D. Gobin, F. Martinez, Melting in rectangular enclosures: experiments and numerical simulations, J. Heat Transfer. 107 (1985). doi:10.1115/1.3247506.

[10] C. Gau, R. Viskanta, Melting and Solidification of a Pure Metal on a Vertical Wall, J. Heat Transfer. 108 (1986) 174. doi:10.1115/1.3246884

[11] C. Benard, D. Gobin, A. Zanoli, Moving boundary problem: heat conduction in the solid phase of a phase-change material during melting driven by natural convection in the liquid, Int. J. Heat Mass Transf. 29 (1986) 1669-1681. doi:10.1016/0017-9310(86)90108-0.

[12] F. Wolff, R. Viskanta, Melting of a Pure Metal From a Vertical Wall, Exp. Heat Transf. 1 (1987) 17-30. doi:10.1080/08916158708946328.

[13] T.A. Campbell, J.N. Koster, Visualization of liquid-solid interface morphologies in gallium subject to natural convection, J. Cryst. Growth. 140 (1994) 414-425. doi:10.1016/0022-0248(94)90318-2.

[14] O. Ben-David, A. Levy, B. Mikhailovich, A. Azulay, 3D numerical and experimental study of gallium melting in a rectangular container, Int. J. Heat Mass Transf. 67 (2013)
260-271. doi:10.1016/j.ijheatmasstransfer.2013.07.058.

[15] M. Bareiss, H. Beer, Experimental investigation of melting heat transfer with regard to different geometric arrangements, Int. Commun. Heat Mass Transf. 11 (1984) 323-333. doi:10.1016/0735-1933(84)90060-5.

[16] H. Shokouhmand, B. Kamkari, Experimental investigation on melting heat transfer characteristics of lauric acid in a rectangular thermal storage unit, Exp. Therm. Fluid Sci. 50 (2013) 201-212. doi:10.1016/j.expthermflusci.2013.06.010

[17] P. Van Buren, R. Viskanta, Interferometric measurement of heat transfer during melting from a vertical surface, J. Heat Mass Transf. 23 (1980) 568-571.

[18] M. Medrano, M.O. Yilmaz, M. Nogués, I. Martorell, J. Roca, L.F. Cabeza, Experimental evaluation of commercial heat exchangers for use as PCM thermal storage systems, Appl. Energy. 86 (2009) 2047-2055.

doi:10.1016/j.apenergy.2009.01.014.

[19] M. Johnson, M. Fiss, T. Klemm, M. Eck, Test and analysis of a flat plate latent heat storage design, in: Energy Procedia, 2013: pp. 662-671. doi:10.1016/j.egypro.2014.10.221.

[20] P.A. Galione, O. Lehmkuhl, J. Rigola, A. Oliva, Fixed-grid numerical modeling of melting and solidification using variable thermo-physical properties - Application to the melting of n-Octadecane inside a spherical capsule, Int. J. Heat Mass Transf. 86 (2015) 721-743.

doi:10.1016/j.ijheatmasstransfer.2015.03.033.

[21] J.J. Jasper, The Surface Tension of Pure Liquid Compounds, J. Phys. Chem. Ref. Data. 1 (1972) 841-1010. doi:10.1063/1.3253106.

[22] J.W. Elder, Laminar free convection in a vertical slot, J. Fluid Mech. 23 (1965) 77. doi:10.1017/S0022112065001246.

[23] P. Jany, A. Bejan, Scaling theory of melting with natural convection in an enclosure, Int. J. Heat Mass Transf. 31 (1988) 1221-1235. doi:10.1016/0017-9310(88)90065-8.

[24] G.K. Batchelor, Heat transfer by free convection across a closed cavity between vertical boundaries at different temperatures, Q. Appl. Math. 12 (1954) 209-233. http://www.jstor.org/stable/43657713.

[25] J. Elder, Turbulent free convection in a vertical slot, J. Fluid Mech. 23 (1965) 99-111.

[26] M. Raffel, C.E. Willert, S. Wereley, J. Kompenhans, Particle Image Velocimetry: A Practical Guide, Springer-Verlag Berlin Heidelberg, 2007. doi:10.1007/978-3-540-72308-0.

[27] R.J. Adrian, J. Westerweel, Particle Image Velocimetry, Cambridge University Press, 2011.

[28] J. Vogel, J. Felbinger, M. Johnson, Natural convection in high temperature flat plate latent heat thermal energy storage systems, Appl. Energy. 184 (2016) 184-196. doi:10.1016/j.apenergy.2016.10.001. 